\title{
Rupioid Psoriasis and Psoriatic Arthritis in a Patient With Skin of Color
}

\author{
Chelsea E. Steele, MD, MPH; Michael Anderson, MD; Jayson Miedema, MD; Donna Culton, MD, PhD
}

\section{PRACTICE POINTS}

- Rupioid psoriasis in skin of color may present a diagnostic challenge for health care providers.

- Rupioid psoriasis may represent a psoriasis variant that is highly responsive to treatment.

To the Editor:

A 49-year-old black woman presented with multiple hyperkeratotic papules that progressed over the last 2 months to circular plaques with central thick black crust resembling eschar. She first noticed these lesions as firm, small, black papules on the legs and continued to develop new lesions that eventually evolved into large, coin-shaped, hyperkeratotic plaques. Her medical history was notable for stage III non-Hodgkin follicular lymphoma in remission after treatment with rituximab, cyclophosphamide, doxorubicin hydrochloride, vincristine sulfate, and prednisone 7 months earlier, and chronic hepatitis B infection being treated with entecavir. Her family history was not remarkable for psoriasis or inflammatory arthritis.

She initially was seen by internal medicine and was started on topical triamcinolone with no improvement of the lesions. At presentation to dermatology, physical examination revealed firm, small, black, hyperkeratotic papules (Figure 1A) and circular plaques with a rim of erythema and central thick, smooth, black crust resembling eschar (Figure 1B). No other skin changes were noted at the time. The bilateral metacarpophalangeal, bilateral proximal interphalangeal, left wrist, and bilateral ankle joints were remarkable for tenderness, swelling, and reduced range of motion. She noted concomitant arthralgia and stiffness but denied fever. She had no other systemic symptoms including night sweats, weight loss, fatigue, malaise, sun sensitivity, oral ulcers, or hair loss. A radiograph of the hand was negative for erosive changes but showed mild periarticular osteopenia and fusiform soft tissue swelling of the third digit. Given the central appearance of eschar in the larger lesions, the initial differential diagnosis included Sweet syndrome, invasive fungal infection, vasculitis, and recurrent lymphoma.

A $4-\mathrm{mm}$ punch biopsy specimen of a representative lesion on the right leg revealed psoriasiform epidermal hyperplasia, parakeratosis, neutrophils in the stratum corneum and spinosum, elongation of the rete ridges, and superficial vascular ectasia, which favored a diagnosis of psoriasis (Figure 2). A periodic acid-Schiff stain was negative for fungal hyphae. Fungal culture, bacterial tissue culture, and acid-fast bacilli smear were negative. Absence of deep dermal inflammation precluded a diagnosis of Sweet syndrome. Further notable laboratory studies included negative human immunodeficiency virus (HIV) antibody, rapid plasma reagin, hepatitis C antibody, and rheumatoid factor.

At follow-up 2 weeks later, the initial lesions were still present, and she had developed new widespread, well-demarcated, erythematous plaques with silver scale along the scalp, back, chest, and abdomen that were more typical of psoriasis. Oil spots were noted on several fingernails and toenails. Based on the clinicopathologic findings, nail changes, and asymmetric inflammatory arthritis, a diagnosis of rupioid psoriasis with psoriatic arthritis (PsA) was established. Treatment with clobetasol ointment $0.05 \%$ twice daily to active lesions was started. Initiation of systemic therapy with a steroid-sparing agent was deferred in anticipation of care coordination with rheumatology, hepatology, and hematology/oncology due to the patient's history of follicular lymphoma and chronic hepatitis B. Although attempts were made to avoid

From the University of North Carolina at Chapel Hill. Dr. Steele is from the School of Medicine, and Drs. Anderson, Miedema, and Culton are from the Department of Dermatology.

The authors report no conflict of interest.

Correspondence: Chelsea E. Steele, MD, MPH, 410 Market St, Ste 400, Chapel Hill, NC 27517 (csteele212@gmail.com). 

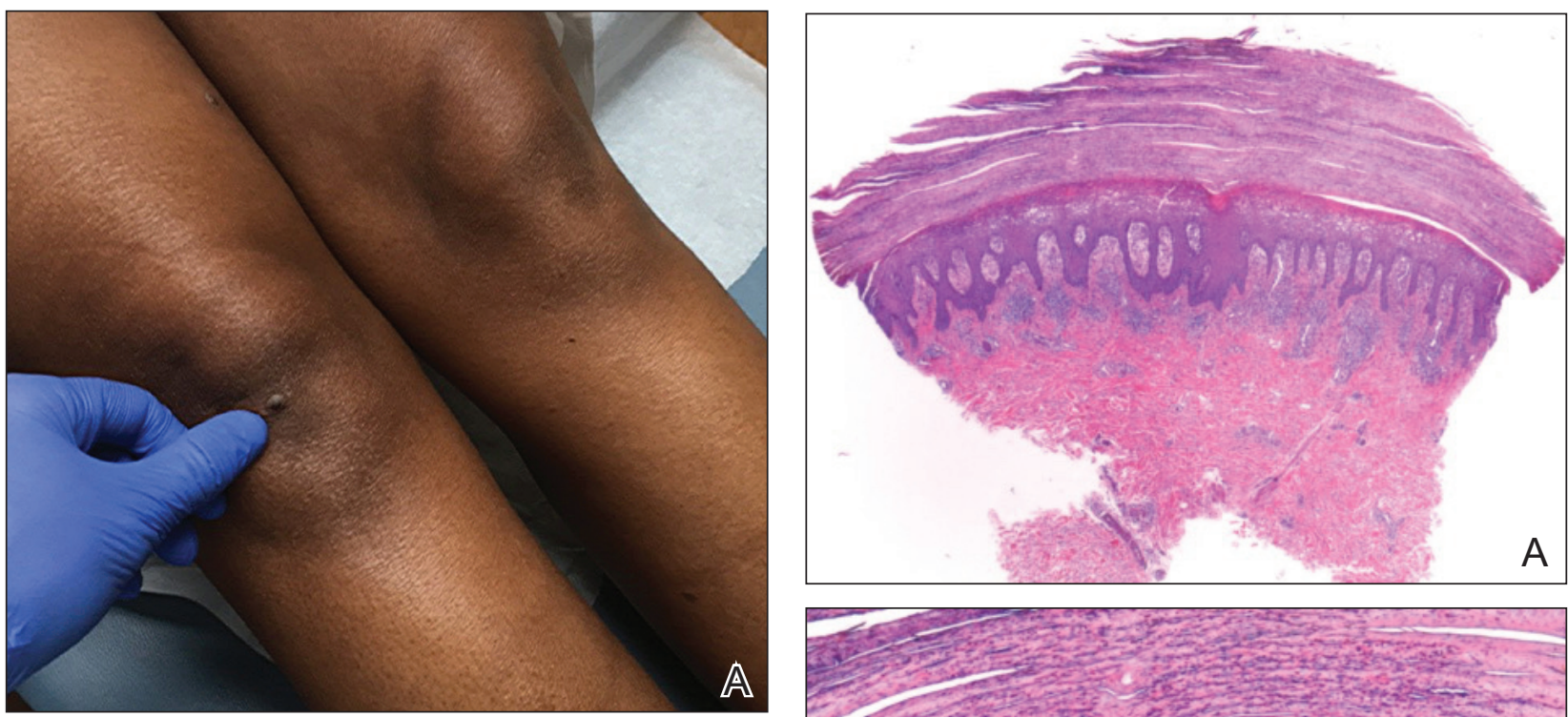

A

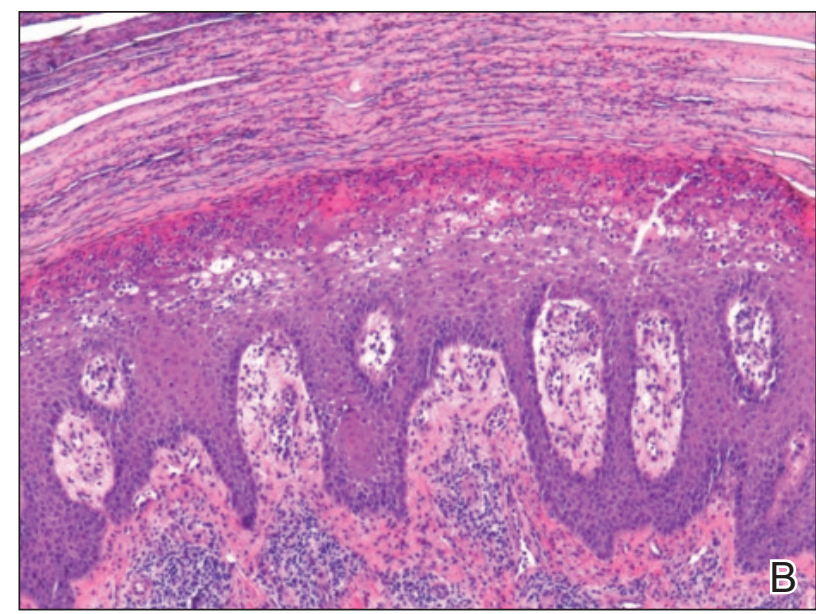

FIGURE 2. A, Histopathology revealed psoriasiform epidermal hyperplasia, parakeratosis, neutrophils in the stratum corneum and spinosum, elongation of the rete ridges, and superficial vascular ectasia $(\mathrm{H} \& \mathrm{E}$, original magnification $\times 20)$. B, Medium-power view of the parakeratosis and vascular ectasia $(H \& E$, original magnification $\times 200)$.

methotrexate $10 \mathrm{mg}$ weekly for 2 weeks followed by titration to $15 \mathrm{mg}$ weekly. Plans were to start a prednisone taper after a month of methotrexate to allow her new treatment time for therapeutic effect. Notably, the patient chose to discontinue prednisone 2 weeks into methotrexate therapy after only two 10-mg doses of methotrexate weekly and well before therapeutic levels were achieved. Despite stopping prednisone early and without a taper, she did not experience a relapse in psoriatic skin lesions. Three months following initiation of methotrexate, she sustained resolution of the cutaneous lesions with only residual postinflammatory hyperpigmentation.

Psoriasis is a common chronic inflammatory skin disorder with multiple clinical presentations. There are several variants of psoriasis that are classified by their morphologic appearance including chronic plaque, guttate, erythrodermic, and pustular, with more than $90 \%$ of cases representing the plaque variant. Less common clinical presentations of psoriasis include rupioid, ostraceous, 
inverse, elephantine, and HIV associated. ${ }^{2}$ Rupioid psoriasis is a rare variant that presents with cone-shaped, limpetlike lesions. ${ }^{3,4}$ Similar to the limited epidemiological and clinical data pertaining to psoriasis in nonwhite racial groups, there also is a paucity of documented reports of rupioid psoriasis in skin of color.

Rupioid comes from the Greek word rhupos, meaning dirt or filth, and is used to describe well-demarcated lesions with thick, yellow, dirty-appearing, adherent crusts resembling oyster shells with a surrounding rim of erythema. ${ }^{5}$ Rupioid psoriasis initially was reported in 1948 and remains an uncommon and infrequently reported variant. ${ }^{6}$ The majority of reported cases have been associated with arthropathy, similar to our patient., Rupioid lesions also have been observed in an array of other diseases, such as secondary syphilis, crusted scabies, disseminated histoplasmosis, HIV, reactive arthritis, and aminoaciduria. ${ }^{7-11}$

Diagnosis of rupioid psoriasis can be confirmed with a skin biopsy, which demonstrates characteristic histopathologic findings of psoriasis. ${ }^{3}$ Laboratory analysis should be performed to rule out other causes of rupioid lesions, and PsA should be differentiated from rheumatoid arthritis if arthropathy is present. In our case, serum rapid plasma reagin, anti-HIV antibody, rheumatoid factor, and fungal cultures were negative. Usin0)g clinical findings, histopathology, laboratory analyses, and radiograph findings, the diagnosis of rupioid psoriasis with PsA was confirmed in our patient.

Psoriasis was not originally suspected in our patient due to the noncharacteristic lesions with smooth black crustsimilar appearing to eschar-and the patient's complicated medical history. Variations in the presentation of psoriasis among white individuals and those with skin of color have been reported in the literature. ${ }^{12,13}$ Psoriatic lesions in darker skin tones may appear more violaceous or hyperpigmented with more conspicuous erythema and thicker plaques. Our patient lacked the classic rupioid appearance of concentric circular layers of dirty, yellow, oysterlike scale, and instead had thick, lamellate, black crust. A PubMed search of articles indexed for MEDLINE using the terms rupioid, coral reef psoriasis, rupioides, and rhupus revealed no other cases of rupioid psoriasis reported in black patients and no cases detailing the variations of rupioid lesions in skin of color. A case of rupioid psoriasis has been reported in a Hispanic patient, but the described psoriatic lesions were more characteristic of the dirty-appearing, conic plaques previously reported. ${ }^{14}$ Our case highlights a unique example of the variable presentations of cutaneous disorders in skin of color and black patients.

Our patient's case of rupioid psoriasis with PsA presented unique challenges for systemic treatment due to her multiple comorbidities. Rupioid psoriasis most often is treated with combination topical and systemic therapy, with agents such as methotrexate and cyclosporine having prior success. ${ }^{3,4}$ This variant of psoriasis is highly responsive to treatment, and marked improvement of lesions has been achieved with topical steroids alone with proper adherence. ${ }^{15}$ Our patient was started on clobetasol ointment $0.05 \%$ while a systemic agent was debated for her PsA. Although she did not have improvement with topical therapy alone, she experienced rapid resolution of the skin lesions after initiation of low-dose prednisone $20 \mathrm{mg}$ daily. Interestingly, our patient did not experience a flare of the skin lesions upon discontinuation of systemic steroids despite the lack of an appropriate taper and methotrexate not having reached therapeutic levels.

The clinical nuances of rupioid psoriasis in skin of color have not yet been described and remain an important diagnostic consideration. Our patient achieved remission of skin lesions with sequential treatment of topical clobetasol, a low-dose systemic steroid, and methotrexate. Based on available reports, rupioid psoriasis may represent a variant of psoriasis that is highly responsive to treatment.

\section{REFERENCES}

1. Mrowietz U, Domm S. Systemic steroids in the treatment of psoriasis: what is fact, what is fiction? I Eur Acad Dermatol Venereol. 2013;27:1022-1025

2. Goldsmith LA, Katz SI, Gilchrest BA, eds. Fitzpatrick's Dermatology in General Medicine. 8th ed. New York, NY: The McGraw-Hill Companies; 2012.

3. Wang JL, Yang JH. Rupioid psoriasis associated with arthropathy. J Dermatol. 1997;24:46-49.

4. Murakami T, Ohtsuki M, Nakagawa H. Rupioid psoriasis with arthropathy. Clin Exp Dermatol. 2000;25:409-412

5. Chung HJ, Marley-Kemp D, Keller M. Rupioid psoriasis and other skin diseases with rupioid manifestations. Cutis. 2014;94:119-121.

6. Salamon M, Omulecki A, Sysa-Jedrzejowska A, et al. Psoriasis rupioides: a rare variant of a common disease. Cutis. 2011;88:135-137.

7. Krase IZ, Cavanaugh K, Curiel-Lewandrowski C. A case of rupioid syphilis. JAAD Case Rep. 2016;2:141-143.

8. Garofalo V, Saraceno R, Milana M, et al. Crusted scabies in a liver transplant patient mimicking rupioid psoriasis. Eur J Dermatol. 2016;26:495-496.

9. Corti M, Villafane MF, Palmieri O, et al. Rupioid histoplasmosis: first case reported in an AIDS patient in Argentina. Rev Inst Med Trop Sao Paulo. 2010;52:279-280.

10. Sehgal VN, Koranne RV, Shyam Prasad AL. Unusual manifestations of Reiter's disease in a child. Dermatologica. 1985;170:77-79.

11. Haim S, Gilhar A, Cohen A. Cutaneous manifestations associated with aminoaciduria. report of two cases. Dermatologica. 1978;156:244-250.

12. McMichael AJ, Vachiramon V, Guzman-Sanchez DA, et al. Psoriasis in African-Americans: a caregivers' survey. J Drugs Dermatol. 2012;11:478-482.

13. Alexis AF, Blackcloud P. Psoriasis in skin of color: epidemiology, genetics, clinical presentation, and treatment nuances. J Clin Aesthet Dermatol. 2014;7:16-24

14. Posligua A, Maldonado C, Gonzalez MG. Rupioid psoriasis preceded by varicella presenting as Koebner phenomenon. J Am Acad Dermatol. 2016;74(5 suppl 1):AB268.

15. Feldman SR, Feldman S, Brown K, et al. "Coral reef" psoriasis: a marker of resistance to topical treatment. J Dermatolog Treat. 2008;19:257-258. 